\title{
Analysis of oscillations in a reciprocally inhibitory network with synaptic depression
}

\author{
Adam L. Taylor Garrison W. Cottrell \\ William B. Kristan, Jr. ${ }^{1}$
}

May 31, 2001

${ }^{1}$ ALT was supported by National Institutes of Health training grant GM08107, and by a La Jolla Interfaces In Science Predoctoral Fellowship (funded by The Burroughs Wellcome Fund). GWC and WBK were supported by National Institutes of Health grant MH43396. We thank Peter Rowat and the two reviewers for their helpful comments and suggestions. 


\begin{abstract}
We present and analyze a model of a two-cell reciprocally inhibitory network that oscillates. The principal mechanism of oscillation is short-term synaptic depression. Using a simple model of depression, and analyzing the system in certain limits, we can derive analytical expressions for various features of the oscillation, including the parameter regime in which stable oscillations occur as well as the period and amplitude of these oscillations. These expressions are functions of three parameters: the time constant of depression, the synaptic strengths, and the amount of tonic excitation the cells receive. We compare our analytical results with the output of numerical simulations, and obtain good agreement between the two. Based on our analysis, we conclude that the oscillations in our network are qualitatively different from those in networks which oscillate due to postinhibitory rebound, spike-frequency adaptation, or other intrinsic (rather than synaptic) adaptational mechanisms. In particular, our network can only oscillate via the synaptic escape mode of Skinner, Kopell and Marder (1994).
\end{abstract}




\section{Background and motivation}

A reciprocally inhibitory (RI) oscillator, also known as a half-center oscillator, is an oscillatory neuronal circuit that consists of two neurons, each of which exerts an inhibitory effect on the other. (More generally, a circuit could be constructed of two populations of neurons, each population inhibiting the other.) An example of such a circuit is shown in Figure 1A. When the circuit oscillates, one cell fires for a time while the other is inhibited below threshold, then the other cell fires for a time while the first is inhibited, and this cycle repeats itself indefinitely. Such oscillators have been found to be important components in a number of central pattern generators (CPGs), including the leech heartbeat CPG (Calabrese, Nadim and Olsen 1995), the Clione swim CPG (Arshavsky, Deliagina, Orlovsky, Panchin, Popova and Sadreyev 1998), the lobster gastric and pyloric CPGs (Selverston and Moulins 1987), the leech swim CPG (Brodfuehrer, Debski, O'Gara and Friesen 1995, Friesen 1989), the Xenopus tadpole swim CPG (Roberts, Soffe and Perrins 1997), and the lamprey swim CPG (Grillner, Deliagina, Ekeberg, el Manira, Hill, Lansner, Orlovsky and Wallén 1995).

A number of mechanisms have been shown to be sufficient to cause an RI circuit to oscillate. Among these are postinhibitory rebound, spike-frequency adaptation, and short-term synaptic depression (Reiss 1962, Perkel and Mulloney 1974). The first two mechanisms are similar, and we will refer to them collectively as intrinsic adaptation. Intrinsic adaptation is a property of the cells themselves, whereas synaptic depression is a property of the connections between them. Without some sort of adaptational mechanism in the circuit, one would not expect it to oscillate. This has in fact been proven in one natural formalization of a non-adaptational RI circuit (Ermentrout 1995).

A number of authors (Wang and Rinzel 1992, Skinner et al. 1994, LoFaro, Kopell, Marder and Hooper 1994, Rowat and Selverston 1997) have analyzed the behavior of intrinsic adaptation RI circuits by using dynamical systems techniques. This work has revealed a number of interesting properties. For instance, Skinner et al. (1994) found that there were at least four functionally distinct modes of oscillation their model could exhibit: intrinsic release, intrinsic escape, synaptic escape, and synaptic release. Each of these modes displayed a different mechanisms of burst termination, and a different frequency response to parameter variations.

Despite the success of applying dynamical systems methods to intrinsic adaptation RI circuits, we are aware of no previous attempt to apply the 
same techniques to RI circuits in which oscillations arise solely due to synaptic depression. Synaptic depression is known to play a role in the oscillation of at least one well-studied RI circuit, the leech heart CPG, for some modes of oscillation (Calabrese et al. 1995). There is also evidence of synaptic depression in CPGs which contain RI subcircuits, including the leech swim CPG (Mangan, Cometa and Friesen 1994) and the lobster pyloric CPG (Manor, Nadim, Abbott and Marder 1997). It has been proposed that synaptic depression acts as a 'switch' in the lobster pyloric CPG, although it is not the ultimate source of oscillation in this system (Nadim, Manor, Kopell and Marder 1999). In addition, there has recently been a great deal of interest in possible functions of synaptic depression in mammalian neocortex (Abbott, Varela, Sen and Nelson 1997, Tsodyks and Markram 1997, Galarreta and Hestrin 1998).

In this paper, we use dynamical systems methods to analyze an RI circuit in which oscillations arise due to synaptic depression. Using these methods, and employing certain approximations, we are able to derive a closed-form expression for the parameter regime in which the model will oscillate. We are also able to derive closed-form expressions for the period, amplitude, and DC offset of these oscillations. We find that these synaptic-depression-mediated oscillations always operate via the synaptic escape mode of Skinner et al. (1994).

The work presented here grew out of a larger effort to understand how the leech swim CPG generates oscillations (Taylor, Cottrell and Kristan 2000). In our earlier work on this subject, we modeled the leech segmental swim CPG as a set of passive cells with instantaneous synapses between them, and connectivity roughly consistent with that determined by experiment. The tentative result of this work was that such a model is able to oscillate, but that even small perturbations of the synaptic strengths destroy this ability. We therefore became interested in the possible role of various cellular and synaptic properties in generating more robust oscillations. One such property, for which there is some evidence in the leech swim CPG (Mangan et al. 1994), is synaptic depression.

While this was our original motivation, we should say at the outset that the purpose of this paper is not to model a known neuronal system, (such as the leech swim CPG). Our goal in this paper is simply to elucidate some of the behaviors one would expect of an RI circuit that oscillates via synaptic depression. It therefore behooves us to study a simple model of such a system, since little data is available to justify various possible embellishments. 
Our model makes predictions about the possible modes of oscillation of such a system, about what parameter regimes permit oscillations, and about how those oscillations should change as the parameters change. Thus we provide a set of hypothetical 'hallmarks' of RI oscillation via synaptic depression. An experimenter could then compare these hallmarks with a system under investigation to evaluate whether it is likely to oscillate via synaptic depression in the way that is described by our model.

\section{Description of the model}

A common simplified model of neuronal dynamics evolves according to the equation

$$
\dot{u}_{i}=-u_{i}+\sum_{j} W_{i j} \sigma\left(u_{j}\right)+b_{i}
$$

There are several ways to derive this equation (or similar equations) from detailed descriptions of neuronal biophysics, all of which involve some simplifying assumptions (Hopfield 1984, Wilson and Cowan 1972). All variables in this equation are unitless, owing to the fact that they are nondimensionalized versions of physical variables. $u_{i}$ corresponds to the membrane potential of cell $i, W_{i j}$ to the maximal postsynaptic current due to the synapse from cell $j$ to cell $i$ (the 'synaptic strength'), $b_{i}$ to any constant current being injected into cell $i . \sigma(x)$ is a sigmoid function as defined by Wilson and Cowan (1972).

We add synaptic depression to this basic model by adding a state variable $d_{j}$ associated with the synapses from cell $j$. This variable ranges from zero to one; at zero the synapse is not depressed at all, and at one the synapse is completely depressed, and can exert no influence on the postsynaptic cell. The modified equation for the dynamics of $u_{i}$ is then given by

$$
\dot{u}_{i}=-u_{i}+\sum_{j}\left(1-d_{j}\right) W_{i j} \sigma\left(u_{j}\right)+b_{i}
$$

We adopt a form for the dynamics of synaptic depression which is first-order and linear:

$$
\tau \dot{d}_{i}=\frac{1}{2} \sigma\left(u_{i}\right)-d_{i}
$$

The parameter $\tau$ is the time constant of synaptic depression. This form for the dynamics of synaptic depression is adopted because it is simple and 
captures the basic qualitative features of the phenomenon (Tsodyks and Markram 1997). The factor of $1 / 2$ in Equation 3 is to ensure that the steadystate synaptic efficacy $\left(\left(1-d_{j}\right) W_{i j} \sigma\left(u_{j}\right)\right.$, where $u_{j}$ has been held constant long enough for $d_{j}$ to come to equilibrium) does not decline with increasing $u_{j}$. (In fact, we can generalize by replacing the factor $1 / 2$ by a factor, $r$, such that $0<r \leq 1 / 2$, and the dynamics do not change qualitatively. We assume $r=1 / 2$ here because this makes the effects of depression large, and for simplicity.) We remain agnostic about the specific biophysics of depression, i.e. whether it arises from presynaptic vesicle depletion, presynaptic $\mathrm{Ca}^{2+}$ channel inactivation, postsynaptic receptor desensitization, or some other mechanism. By using a single time constant, we are implicitly assuming that the time constants associated with onset and offset of depression are identical, another simplifying assumption.

Since we are concerned here only with two-cell networks with symmetric reciprocal inhibition and no self-connections (Figure 1A), we can rewrite the system equations as

$$
\begin{aligned}
\dot{u}_{1} & =-u_{1}-\left(1-d_{2}\right) W \sigma\left(u_{2}\right)+b \\
\dot{u}_{2} & =-u_{2}-\left(1-d_{1}\right) W \sigma\left(u_{1}\right)+b \\
\tau \dot{d}_{1} & =\frac{1}{2} \sigma\left(u_{1}\right)-d_{1} \\
\tau \dot{d_{2}} & =\frac{1}{2} \sigma\left(u_{2}\right)-d_{2}
\end{aligned}
$$

Here $W$ is taken to be nonnegative, since we are interested only in inhibitory networks. Thus the model has just three parameters: $W, b$, and $\tau$.

For a range of parameter settings, the two-cell system with synaptic depression is capable of stable oscillation, as shown in Figure 1B. The sigmoid function used here and in later examples is $\sigma(x)=\operatorname{logistic}(4 x)=1 /\left(1+e^{-4 x}\right)$. The factor of four is to make the slope at $x=0$ equal to one, and thus make the linear regime approximately one unit wide. The oscillation proceeds in the following manner: Beginning with cell 1 above threshold and cell 2 below (marked as interval a in the Figure), cell 1 inhibits cell 2, and the membrane potential of cell 1 remains relatively constant. This inhibition wanes, however, due to a buildup of synaptic depression $\left(d_{1}\right)$, so the membrane potential of cell 2 creeps slowly upwards. At the same time, the synapse from cell 2 onto cell 1 is recovering (reflected by the decrease in $d_{2}$ ) while cell 2 is below threshold. Eventually, cell 2's membrane potential is high enough to cross threshold and inhibit cell 1 (interval b). Following this quick transition, we arrive at a state in which cell 2 is above threshold and cell 1 is below (interval 

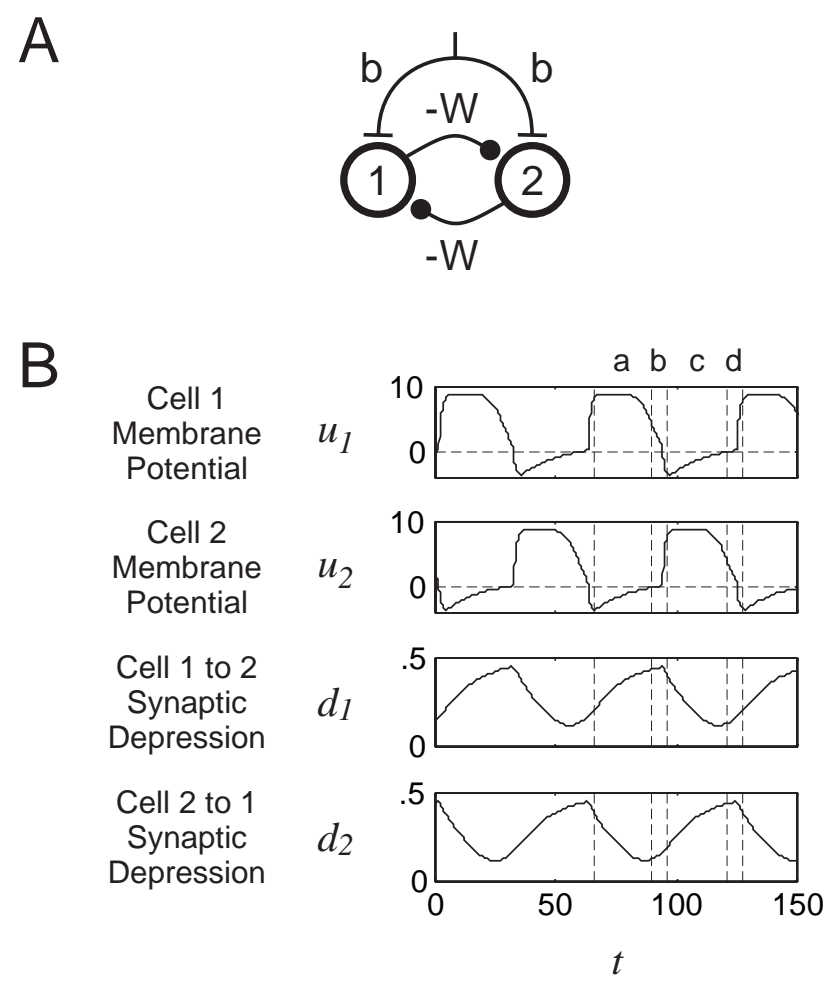

Figure 1: Circuit schematic and example oscillations of the system. In Panel A, bars represent excitatory synapses and circles represent inhibitory synapses. In Panel B, the values of the four state variables of the system, $u_{1}$, $u_{2}, d_{1}$, and $d_{2}$, are shown versus time, $t$. All state variables are unitless, owing to the fact that they are nondimensionalized versions of physical variables. $u_{i}$ values are membrane potentials measured relative to the potential at which the synapses are half-activated, this quantity being given in units of the width of the linear regime of the synaptic activation curve. Time is in units of the membrane time constant of the cells. The parameters for the simulation in Panel B are $W=16, b=9, \tau=16$, with $\sigma(x)=\operatorname{logistic}(4 x)=1 /\left(1+e^{-4 x}\right)$. See the text for an explanation of the intervals labelled a, b, c, and d. 
c). This situation is the mirror image of the original state, with the roles of the cells reversed. Thus the membrane potential of cell 1 creeps slowly upward as the incoming inhibition wanes, until cell 1 crosses threshold and begins to inhibit cell 2. Again, a switch quickly occurs (interval d), bringing cell 1 above threshold and pushing cell 2 below. This is the original state, and so the process repeats itself, and an oscillation is produced.

\section{Analysis of model behavior}

In this section we analyze the system to understand in detail how the oscillation 'works'. This enables us to derive the range of parameters for which the system oscillates, and how the features of that oscillation (period, amplitude, etc.) vary as the parameters are varied. Our analysis relies on two simplifications:

1. Synaptic depression changes slowly relative to changes in membrane potential $(\tau \gg 1)$

2. The sigmoid function can be approximated as a unit step function $(\sigma(x) \approx s(x)$, where $s(x)=1$ if $x \geq 0$, otherwise $s(x)=0)$

Using these two simplifications will allow us to derive approximate closedform expressions for the parameter regime in which oscillations are possible and for the period and amplitude of these oscillations. The first allows us to apply singular perturbation theory, considering separately the 'fast' $u$ system of Equations 4 and 5 and the 'slow' $d$ system of Equations 6 and 7. Furthermore, synaptic depression seems to be about 5-10 times slower than typical membrane time constants in many systems of interest, among them the leech swim CPG (Mangan et al. 1994, Calabrese et al. 1995, Manor et al. 1997). The second simplification allows us to replace a difficult-to-analyze nonlinear function with an easier-to-analyze piecewise constant function. This also seems to accord roughly with the behavior of known RI circuits: Cells oscillate between a hyperpolarized level at which they have little synaptic output, and a highly active level at which they have substantial synaptic output (Friesen 1989, Calabrese et al. 1995, Manor et al. 1997). Making these two assumptions results in a reduced system that is piecewise linear, and thus can be solved exactly. 


\section{The fast system}

Assumption 1 implies that the $u$ variables will come to equilibrium much faster than the $d$ variables, and will remain at approximate equilibrium as the $d$ variables slowly evolve. If the $u$ variables are approximately at equilibrium, then $\dot{u}_{1} \approx \dot{u}_{2} \approx 0$. Thus Equations 4 and 5 become

$$
\begin{aligned}
& -u_{1}-\left(1-d_{2}\right) W \sigma\left(u_{2}\right)+b \approx 0 \\
& -u_{2}-\left(1-d_{1}\right) W \sigma\left(u_{1}\right)+b \approx 0
\end{aligned}
$$

We would like to solve these equations for $u_{1}$ and $u_{2}$ given $d_{1}$ and $d_{2}$, but the nonlinearity makes it impossible to do this exactly, so we invoke assumption 2 , replacing the sigmoid with a unit step function. Thus Equations 8 and 9 become

$$
\begin{aligned}
& -u_{1}-\left(1-d_{2}\right) W s\left(u_{2}\right)+b \approx 0 \\
& -u_{2}-\left(1-d_{1}\right) W s\left(u_{1}\right)+b \approx 0
\end{aligned}
$$

Since the step function can only take on the values 0 or 1 , we can break down Equations 10 and 11 into four separate cases, corresponding to the four possible values of the pair $\left(s\left(u_{1}\right), s\left(u_{2}\right)\right)$. In each of these cases we can then solve exactly for the $u$ equilibrium.

Case $00 s\left(u_{1}\right)=0, s\left(u_{2}\right)=0$ :

In this case $u_{1}<0$ and $u_{2}<0$, and we have

$$
\begin{aligned}
& u_{1}=b<0 \\
& u_{2}=b<0
\end{aligned}
$$

In this case, the inequalities imply that this equilibrium of the $u$ system only exists if $b<0$. This makes intuitive sense: $b<0$ corresponds to the situation in which the tonic input is insufficient to elevate the cells' membrane potential above synaptic threshold.

Case $01 s\left(u_{1}\right)=0, s\left(u_{2}\right)=1$ :

In this case $u_{1}<0$ and $u_{2}>0$, and we have

$$
\begin{aligned}
& u_{1}=b-\left(1-d_{2}\right) W<0 \\
& u_{2}=b>0
\end{aligned}
$$


Note that the inequalities imply that this solution exists only if $b>0$, and then only if the the values of $d_{2}, b$, and $W$ satisfy the inequality in Equation 14. We can reexpress this inequality as $d_{2}<1-b / W$. Again, this makes intuitive sense: cell 2 is above threshold and cell 1 below, which can happen only if the tonic input is sufficiently excitatory and the inhibition onto cell 1 is not too depressed, i.e. only if $d_{2}$ is below some specified value. Note that the inequality in Equation 14 implies that $b>0$, since $d_{2}$ must lie in the interval $[0,1 / 2]$.

Case $10 s\left(u_{1}\right)=1, s\left(u_{2}\right)=0$ :

This case is just the mirror image of the above case. In this case $u_{1}>0$ and $u_{2}<0$, and we have

$$
\begin{aligned}
& u_{1}=b>0 \\
& u_{2}=b-\left(1-d_{1}\right) W<0
\end{aligned}
$$

As in the above case, the inequality in Equation 17 can be reexpressed as $d_{1}<1-b / W$. Again, this implies that $b>0$.

Case $11 s\left(u_{1}\right)=1, s\left(u_{2}\right)=1$ :

In this case $u_{1}>0$ and $u_{2}>0$, and we have

$$
\begin{aligned}
& u_{1}=b-\left(1-d_{2}\right) W>0 \\
& u_{2}=b-\left(1-d_{1}\right) W>0
\end{aligned}
$$

As in cases 01 and 10, the inequalities can be reexpressed as

$$
\begin{aligned}
& d_{1}>1-b / W \\
& d_{2}>1-b / W
\end{aligned}
$$

Intuitively, this case corresponds to both synapses being so depressed that neither cell is able to inhibit the other below threshold, so both cells are active. And again, it should be noted that the inequalities in this case imply that $b>0$.

In addition to deriving solutions for the equilibria of the $u$ system, we have also derived the conditions under which each exists, in terms of the $W, b, d_{1}$ and $d_{2}$. Furthermore, for each case above there is a unique $u$ equilibrium, 
so below we will often speak, for instance, of 'the $\mathbf{0 1}$ equilibrium' or 'the $\mathbf{1 1}$ equilibrium'.

The regions in the $\left(d_{1}, d_{2}\right)$ plane where each equilibrium is possible are shown in Figure 2. Figure 2A shows the rather dull situation which results when $b<0$. In this case, only the $\mathbf{0 0}$ equilibrium is possible; both cells are inactive because they receive no tonic excitation. For $b>0$, as shown in Figures $2 \mathrm{~B}$ and $2 \mathrm{C}$, the picture is more interesting. The lines $d_{1}=1-b / W$ and $d_{2}=1-b / W$ divide the plane into four 'quadrants', each of which allows for a different set of $u$ equilibria, from the case-by-case analysis above. Only one equilibrium is possible in three of these quadrants, but in the fourth (the lower left one) two equilibria are possible: the $\mathbf{0 1}$ and $\mathbf{1 0}$ equilibria. This is because $d_{1}<1-b / W$ defines the region of possible $\left(d_{1}, d_{2}\right)$ values for the 10 equilibrium (we will call points in this region viable, and the region as a whole the viable region for that equilibrium), and $d_{2}<1-b / W$ defines the viable region for the $\mathbf{0 1}$ equilibrium, and the lower left quadrant in Figure 2B is simply the overlap of these two regions.

Figure $2 \mathrm{~B}$ represents the case where $b / W>1 / 2$, and thus $1-b / W<1 / 2$. In this case the lines $d_{1}=1-b / W$ and $d_{2}=1-b / W$ fall within the square of possible $\left(d_{1}, d_{2}\right)$ values (from Equation 3 , each of the $d_{i}$ 's must be on the interval $[0,1 / 2])$. If $b / W<1 / 2$, these lines fall outside the square, and the system no longer has any regions with only one possible $u$ equilibrium. The system can be in either the $\mathbf{0 1}$ or $\mathbf{1 0}$ equilibria, regardless of $\left(d_{1}, d_{2}\right)$, and

it must be in one of these two equilibria. This has important consequences for the overall behavior of the system, as will become clear when we consider the behavior of the slow $d$ system.

\section{The slow system}

Now that we have characterized the equilibria of the fast $u$ system for given $d_{1}$ and $d_{2}$, we would like to describe how the slow $d$ variables evolve, given that the $u$ system is maintained at equilibrium. In Equations 6 and 7, $u_{1}$ and $u_{2}$ appear only via $\sigma\left(u_{1}\right)$ and $\sigma\left(u_{2}\right)$. Utilizing assumption 2 , we can write the slow dynamics as

$$
\begin{aligned}
\tau \dot{d}_{1} & =\frac{1}{2} s\left(u_{1}\right)-d_{1} \\
\tau \dot{d}_{2} & =\frac{1}{2} s\left(u_{2}\right)-d_{2}
\end{aligned}
$$

Each of the $u$ equilibria discussed above corresponds to one of the four possible values of $\left(s\left(u_{1}\right), s\left(u_{2}\right)\right)$. Which $u$ equilibrium the system currently oc- 



Figure 2: The regions of the $\left(d_{1}, d_{2}\right)$ plane for which the various $u$ equilibria exist. Each panel shows the $\left(d_{1}, d_{2}\right)$ plane for a different parameter regime. The dashed lines in each panel indicate the square to which $\left(d_{1}, d_{2}\right)$ is constrained. For Panel A, $b<0$ and only $u$ equilibrium $\mathbf{0 0}$ is possible. Panel B represents the situation in which $b>0$ and $b / W>1 / 2$. In this case there are three possible $u$ equilibria. Panel $\mathrm{C}$, for which $b>0$ and $b / W<1 / 2$, is similar to Panel $\mathrm{B}$, except that the lines $d_{1}=1-b / W$ and $d_{2}=1-b / W$ are now outside the square of possible $\left(d_{1}, d_{2}\right)$ values. 
cupies will determine the dynamics of the $d$ system. Because of this, we can draw a separate phase portrait in the $\left(d_{1}, d_{2}\right)$ plane for each of these equilibria. This is done in Figure 3. In general, the $d$ dynamics have a single fixed point, which is stable, at $\left(s\left(u_{1}\right) / 2, s\left(u_{2}\right) / 2\right)$. However, for $u$ equilibria $\mathbf{0 1}$ and 10, this point may or may not be viable, depending on whether $b / W>1 / 2$ or not, i.e. depending on whether Figure $2 \mathrm{~B}$ or $\mathrm{C}$ applies. We concentrate here on the case depicted in Figure 2B, for which the $d$ fixed points for the 01 and 10 equilibria are not in the respective viable regions. Accordingly, Figure 3 corresponds with same range of parameters as Figure $2 \mathrm{~B}$, in that $1-b / W>1 / 2$ in the Figure.

For $b>0$ and $b / W>1 / 2$, the $\mathbf{0 1}$ and $\mathbf{1 0}$ equilibria are not in the viable region. (Equivalently, we could just say 'For $b / W>1 / 2 \ldots$, , since this implies $b>0$ given our assumption that $W>0$.) In this case the $d$ system will move toward the fixed point until it hits the edge of the viable region, where the $u$ equilibrium the system has been 'tracking' will disappear. At this point $\left(u_{1}, u_{2}\right)$ will quickly (i.e. on the time scale of the fast system) change to some other equilibrium of the fast $u$ system. Note that at all points where the $d$ system can cross from a viable region to a nonviable region, there is only one $u$ equilibrium to which the fast system can settle after the transition. Thus the post-transition $u$ equilibrium is uniquely determined in all cases. Graphically, the current value of $\left(d_{1}, d_{2}\right)$ will move within the white region of one of the panels of Figure 3 until it hits a viable/nonviable (white/dark gray) border, at which point it will 'jump' to the same $\left(d_{1}, d_{2}\right)$ point on another panel, the destination panel being that one for which the current value of $\left(d_{1}, d_{2}\right)$ is in the viable region. Intuitively, the jumps happen when one of the synapses depresses enough (or recovers enough) that the system as a whole can no longer support the $u$-equilibrium the system was previously in. For instance, if the system is in the 10-equilibrium, but $\left(d_{1}, d_{2}\right)$ crosses the line $d_{1}=1-b / W, d_{1}$ will now be too large for cell 1 to inhibit cell 2 below threshold, and the system will quickly jump to a state with cell 2 above threshold.

\section{Attractors}

Since the reduced dynamics of $\left(d_{1}, d_{2}\right)$ are piecewise linear, they can be solved exactly. The general solution (including transients) is cumbersome, however, and does not yield additional insight. Instead of presenting the general solution, we describe the various steady-state solutions of the system. It should 


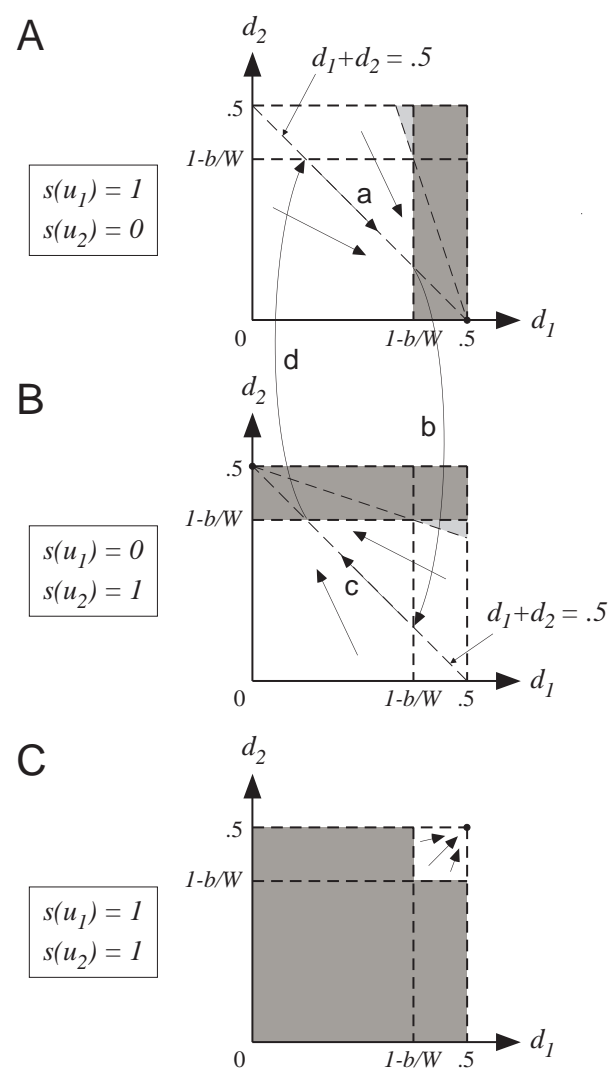

Figure 3: The viable regions and phase portraits for the three $u$ equilibria possible when $b / W>1 / 2$. Points which represent viable states of the system are in white, nonviable points are dark gray. Example trajectories for each $u$ equilibrium are shown. The system as shown contains two attractors: a stable fixed point at $s\left(u_{1}\right)=1, s\left(u_{2}\right)=1, d_{1}=1 / 2, d_{2}=1 / 2$ and a stable limit cycle which is comprised of line segments along the line $d_{1}+d_{2}=1 / 2$ for $s\left(u_{1}\right)=1, s\left(u_{2}\right)=0$ and $s\left(u_{1}\right)=0, s\left(u_{2}\right)=1$. The points in the limit cycle where the $u$ equilibrium changes are indicated by the arcs connecting the $s\left(u_{1}\right)=1, s\left(u_{2}\right)=0$ and $s\left(u_{1}\right)=0, s\left(u_{2}\right)=1$ planes (Panels $\mathrm{A}$ and $\mathrm{B}$, respectively). The various segments of the limit cycle are also labelled $\mathrm{a}, \mathrm{b}, \mathrm{c}$, and d, to correspond with the labelled parts of the oscillation in Figure 1. The light gray triangles represent regions for which the system will not oscillate, but rather will eventually transition to the $\mathbf{1 1}$ equilibrium. 
be noted that these solutions are exact for the reduced system only, and are only approximate solutions of the full system. We assess how good these approximations are in the "Comparison with Simulations" section below.

\section{Limit cycles}

For certain values of the parameters, the system is capable of oscillation, and the mechanism of oscillation can be visualized in Figure 3. For the 10 equilibrium (Figure $3 \mathrm{~A})$, the dynamics of $\left(d_{1}, d_{2}\right)$ has an attracting fixed point at $(1 / 2,0)$. Therefore $\left(d_{1}, d_{2}\right)$ moves towards this point but is unable to reach it because it is in the nonviable region. Upon reaching the line $d_{1}=$ $1-b / W,\left(s\left(u_{1}\right), s\left(u_{2}\right)\right)$ switches to the value $(0,1)$. (Actually, if $d_{2}>1-b / W$ at the transition, $\left(s\left(u_{1}\right), s\left(u_{2}\right)\right)$ switches instead to $(1,1)$, and no oscillation occurs. We discuss this possibility below, but for now assume that $d_{2}<1-$ $b / W$ at the transition.) For $\left(s\left(u_{1}\right), s\left(u_{2}\right)\right)=(0,1)$ (Figure $\left.3 \mathrm{~B}\right)$, the dynamics of $\left(d_{1}, d_{2}\right)$ have a attracting fixed point at $(0,1 / 2)$, and $\left(d_{1}, d_{2}\right)$ begins to move towards this point. Once again the nonviable region intervenes, causing a switch back to $\left(s\left(u_{1}\right), s\left(u_{2}\right)\right)=(1,0)$. This cycle then repeats itself.

The fact that this cyclic behavior approaches a stable limit cycle can be seen by considering the quantity $d_{1}+d_{2}$. Adding Equation 22 to Equation 23 we have that

$$
\frac{d}{d t}\left(d_{1}+d_{2}\right)=\frac{1}{2}\left[s\left(u_{1}\right)+s\left(u_{2}\right)\right]-\left(d_{1}+d_{2}\right)
$$

Since $s\left(u_{1}\right)+s\left(u_{2}\right)=1$ for both $\left(s\left(u_{1}\right), s\left(u_{2}\right)\right)=(1,0)$ and $\left(s\left(u_{1}\right), s\left(u_{2}\right)\right)=$ $(0,1)$, the sum $d_{1}+d_{2}$ monotonically approaches $1 / 2$ as the system switches back and forth between the $u$ equilibria, and thus the system approaches a stable limit cycle along the line $d_{1}+d_{2}=1 / 2$. This limit cycle is shown in Figures $3 \mathrm{~A}$ and $3 \mathrm{~B}$. The various phases of the limit cycle are labelled a, b, c, and $d$ in the Figure to correspond with the same labels in Figure 1.

Since the reduced dynamics are linear between the jump points, we can write an exact expression for the steady-state oscillatory solution:

$$
\begin{aligned}
& d_{1}(t)= \begin{cases}d_{\mathrm{hi}} \exp \left(-t_{\varphi} / \tau\right) & t_{\varphi} \leq T / 2 \\
1 / 2+\left(d_{\mathrm{lo}}-1 / 2\right) \exp \left(-t_{\varphi} / \tau\right) & t_{\varphi}>T / 2\end{cases} \\
& d_{2}(t)= \begin{cases}1 / 2+\left(d_{\mathrm{lo}}-1 / 2\right) \exp \left(-t_{\varphi} / \tau\right) & t_{\varphi} \leq T / 2 \\
d_{\mathrm{hi}} \exp \left(-t_{\varphi} / \tau\right) & t_{\varphi}>T / 2\end{cases}
\end{aligned}
$$


where $t_{\varphi}=\bmod (t, T), d_{\mathrm{hi}}=1-b / W, d_{\mathrm{lo}}=1-d_{\mathrm{hi}}=b / W-1 / 2$, and $T$ is the period of the oscillation, given below. This is the steady-state solution for the initial conditions $d_{1}(0)=d_{\mathrm{hi}}$ and $d_{2}(0)=d_{\mathrm{lo}}$, but any initial conditions such that $d_{1}+d_{2}=1 / 2$ will also yield a steady-state oscillation, which will simply be a phase-shifted version of the above. Essentially, the $d_{i}$ 's exponentially decay from $d_{\mathrm{hi}}$ to $d_{\mathrm{lo}}$ and back again, in antiphase to one another.

The period of the oscillations is twice the time it takes $\left(d_{1}, d_{2}\right)$ to go from one jump point to the other along the line $d_{1}+d_{2}=1 / 2$, thus we can show that the period is equal to

$$
T=-2 \tau \ln \left[\frac{1}{2(1-b / W)}-1\right]
$$

From Equations 25 and 26 (or by examining the geometry of the limit cycle, as shown in Figures $3 \mathrm{~A}$ and $3 \mathrm{~B}$ ) we can derive the amplitude (defined here as the difference between the maximum and minimum values) and timeaverage of the oscillations of the $d$ variables:

$$
\begin{aligned}
A_{d} & =\frac{3}{2}-2 \frac{b}{W} \\
\langle d\rangle & =\frac{1}{4}
\end{aligned}
$$

Similarly, we can derive these quantities for the $u$ variables:

$$
\begin{aligned}
& A_{u}=\frac{3}{2} W-b \\
& \langle u\rangle=b-\frac{1}{4} W+\frac{\tau}{T}(b-W)\left[1-\exp \left(-\frac{T}{2 \tau}\right)\right]
\end{aligned}
$$

\section{Equilibrium points}

In addition to the stable limit cycle, the system also allows for stable fixed points. A stable fixed point is located at $\left(d_{1}, d_{2}\right)=(1 / 2,1 / 2)$ for the 11 equilibrium (Figure 3C). This corresponds to the situation in which both synapses are maximally depressed, and neither synapse is able to inhibit the postsynaptic cell below threshold. In fact all states for which the $u$ system is at the $\mathbf{1 1}$ equilibrium are in the basin of attraction for this fixed point, so if both $d_{1}$ and $d_{2}$ are greater than $1-b / W$ at any point in time, the system settles to this fixed point. This reflects the fact that if both synapses get so depressed that neither cell is able to inhibit the other below threshold, then both synapses can only get increasingly depressed as time goes on, and hence approach a state of maximal depression, represented by the fixed point. 
Points in the other $u$ equilibria that are also in the basin of attraction for this fixed point are shown in light gray in Figures $3 \mathrm{~A}$ and $3 \mathrm{~B}$.

All of the above applies to the $b / W>1 / 2$ case. On the other hand, if $0<b / W<1 / 2$, as in Figure $2 \mathrm{C}$, neither the limit cycle or the described stable fixed point exist. In this case the $d$ equilibria in Figures $3 \mathrm{~A}$ and $3 \mathrm{~B}$ are in the viable region, and are therefore equilibria of the full system. The system behaves very much like a RI circuit without synaptic depression: It possesses two mirror-symmetric stable states, one with cell 1 above threshold and cell 2 below, and one with cell 2 above and cell 1 below.

\section{Oscillatory regime}

The size of the viable region for the $\mathbf{1 1}$ equilibrium changes as a function of the parameters, since its borders are defined by the lines $d_{1}=1-W / b$ and $d_{2}=1-W / b$. In contrast, the limit cycle (when it exists) is always on the line $d_{1}+d_{2}=1 / 2$. As a consequence, the limit cycle exists only for parameter values such that the valid region of the $\mathbf{1 1}$ equilibrium does not overlap the line $d_{1}+d_{2}=1 / 2$. This leads to the constraint that $1-b / W>1 / 4$ (or, equivalently, $b / W<3 / 4)$ in order for a stable limit cycle to exist, since the corner of the viable region for the $\mathbf{1 1}$ equilibrium is at $\left(d_{1}, d_{2}\right)=(1-b / W, 1-$ $b / W)$, and this point just intersects the limit cycle when $1-b / W=1 / 4$. Combined with the fact that $b$ must also satisfy $b / W>1 / 2$ for the limit cycle to exist, we have the following constraint which must be satisfied for the system to oscillate:

$$
\frac{1}{2}<b / W<\frac{3}{4}
$$

This defines the region of parameter space in which the system possesses a limit cycle attractor.

\section{Comparison with simulations}

The analysis above applies in the limits of large $\tau$ and steep sigmoid function. However, if 'typical' values of the $u_{i}$ 's are such that $\left|u_{i}\right| \gg 1$, then even if the linear regime of the sigmoid is of approximately unit width, the sigmoids will be saturated most of the time, and so will be effectively steep. In the case of oscillations, therefore, we would expect our approximations to be most accurate when the amplitude of the oscillations is much greater than one. In simulations, we find that the oscillations get bigger in amplitude as $W$ and 
$b$ get bigger. (This is fully consistent with Equation 30: if we scale both $W$ and $b$ by a constant factor, $A_{u}$ will be scaled by the same factor.) Thus we would expect our approximations give the best agreement with simulations of the full system for large $\tau, W$, and $b$.

As a practical matter, however, we find that the results of numerical simulations of the full system agree fairly well with the analytical predictions, even when the system is far from these limits. Figure 4 shows the volume of parameter space for which the model of Equations 4-7 is oscillatory, both in numerical simulations and according to the analysis. In general, the analysis gives a good approximation to the oscillatory regime of the full system. Of course, the closer the system parameters adhere to the assumptions, the better the predictions are: For large $\tau$ the area of the $(W, b)$ plane which is oscillatory in simulation is closest to the theoretical prediction, with gradually lessening agreement for lower $\tau$.

To further illustrate the qualitative agreement between simulation and analysis, Figure 5 shows the period of the system as a function of $b$, for a number of discrete values of $W$ and $\tau$. The analytical value given by Equation 27 shows good agreement with the measured period from numerical simulations, over a wide range of parameter values. The agreement is again best for large values of $\tau$, as would be expected. Also, the agreement is better for large values of $W$. As $W$ is decreased, Assumption 2 becomes less appropriate, and the agreement between analysis and numerical experiment is correspondingly worse. Nonetheless, the agreement between analysis and simulation is quite respectable even in Panel $\mathrm{C}$ of Figure 5, when $W$ is only a few times greater than one.

\section{Discussion}

We have demonstrated an extremely simple RI circuit that oscillates solely due to synaptic depression. We have analyzed the circuit to elucidate the mechanisms of this oscillation. Using simple approximations, we have derived closed form expressions for the region in parameter space in which oscillation occurs, and for various characteristics of this oscillation. Furthermore, we have shown that these approximate expressions match simulations of the full system for a wide range of parameter values. 

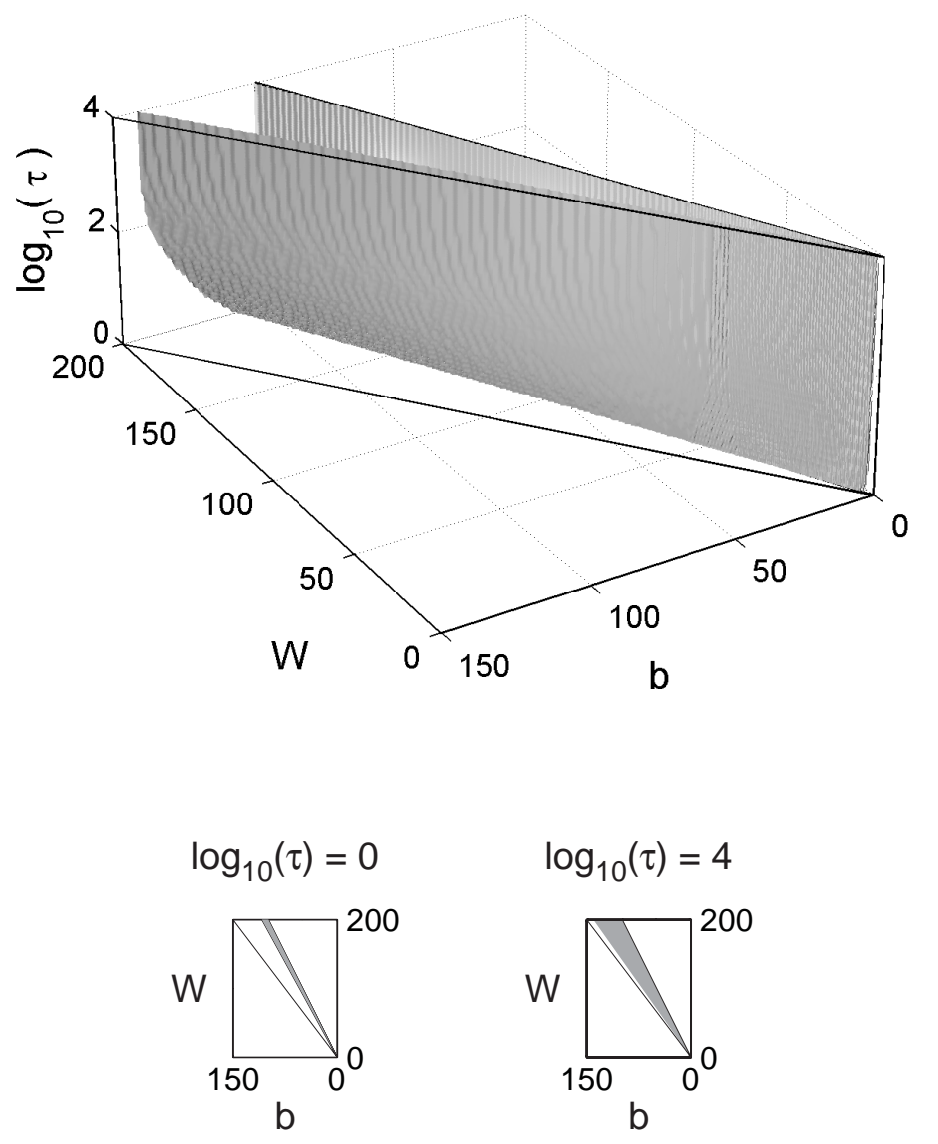

Figure 4: The volume of parameter space for which the system oscillates. Systems which produce stable oscillations in numerical simulations of the full system are on the inside of the gray 'wedge'. The planes $b / W=1 / 2$ and $b / W=3 / 4$, which delineate the analytically determined oscillatory regime (Equation 32), are also shown, in outline, by solid lines. A log scale is used for the $\tau$ axis to display a large range of possible values. The insets show the oscillatory regime in the $(W, b)$ plane for fixed values of $\tau$, i.e. horizontal slices through the three-dimensional volume. The gray region in the insets is the interior of the wedge shown in the three-dimensional view. 


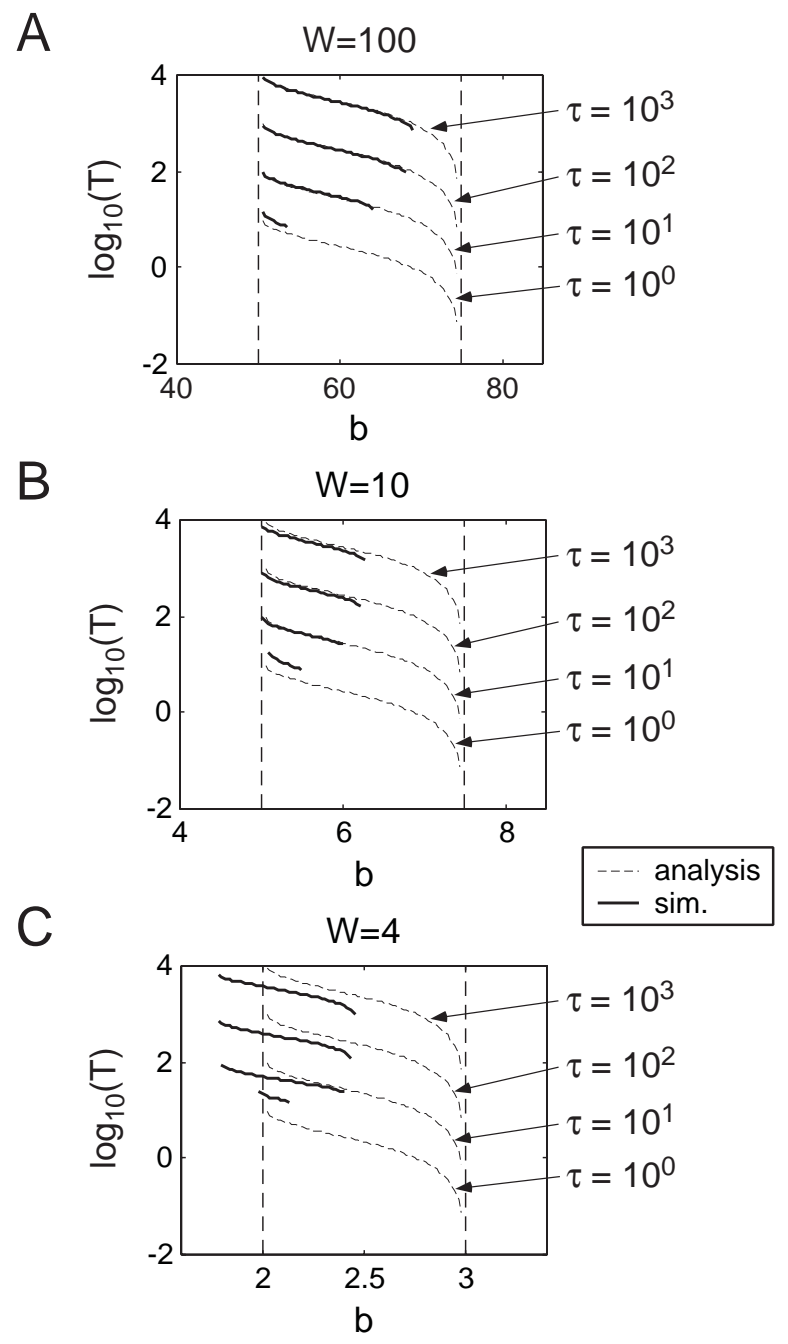

Figure 5: The period, $T$, of the oscillation as a function of $b$ for various values of $W$ and $\tau$. This figure shows the agreement between the analytical value for the period of the oscillation (Equation 27) and the results of numerical simulations of the full four-dimensional system. A log scale is used for the $T$ axis to display periods over several orders of magnitude conveniently. The dashed lines indicate the limits of the oscillatory regime according to the analysis. The curves end where the system no longer oscillates, in simulation or according to the analysis. 


\section{Synaptic depression vs. intrinsic adaptation}

A natural question to ask is whether oscillations in our system differ in any qualitative way from oscillations arising due to intrinsic cellular properties. On the face of it, we might expect the two mechanisms to lead to similar behavior. If we consider only the input-output relationship of a single neuron, intrinsic adaptation and synaptic depression seem very similar in their effects. In response to a step increase in input, both intrinsic adaptation and synaptic depression produce a similar effect on the output: a large transient increase in output followed by a decay to a level intermediate between the initial level and the peak transient level. This similarity is misleading, however. The oscillations in our system $d o$ differ qualitatively from those arising in intrinsic adaptation circuits. In order to describe the ways in which they differ, we will first discuss the modes of oscillation which arise in intrinsic adaptation RI networks, and then contrast these with the single mode of oscillation available to our system.

Skinner et al. (1994), building on earlier work by Wang and Rinzel (1992), analyzed oscillations of RI networks in which the model cells were of the Morris-Lecar type. These cells include a fast $\mathrm{Ca}^{2+}$ current as well as a slow $\mathrm{K}^{+}$current. (Actually, their analysis does not depend on the particular ionic currents present, but only on the nullcline structure of the dynamics. The Morris-Lecar cell is simply a well- known model with the required nullcline structure.) They found that such a model circuit can support oscillation in one of four distinct modes: intrinsic escape, intrinsic release, synaptic escape, and synaptic release. Which mode the system is in is characterized by the events leading up to burst termination, and each mode has particular features, such as whether the period of oscillation is sensitive to the synaptic threshold or not. The two 'intrinsic' modes of oscillation are made possible by the presence of the fast $\mathrm{Ca}^{2+}$ current, which makes the membrane bistable on short time scales. When this current is removed, oscillation can still occur, but only via the two 'synaptic' mechanisms (A.L. Taylor, unpublished simulations).

In the synaptic escape mode of oscillation, the inhibited cell's membrane potential creeps upward until it crosses the synaptic threshold. At this point, a fast switch occurs in which the inhibited cell becomes dominant and viceversa. When the dominant/inhibited transitions take place in this fashion, it is found that the frequency of oscillations increases as the synaptic threshold is lowered. In the synaptic release mode, the dominant cell's membrane 
potential creeps downward until it crosses the synaptic threshold, at which point the dominant/inhibited switch occurs. In this case, it is found that the frequency of oscillations decreases as the synaptic threshold is lowered. Since it is possible for the membrane potential of the dominant cell to creep downward at the same time as the membrane potential of the inhibited cell is creeping upward, the mode in which the system oscillates will be determined by which cell's membrane potential 'hits' the synaptic threshold first.

In our system, on the other hand, only the synaptic escape mode of oscillation occurs. The membrane potential of the inhibited cell creeps upward due to the waning synaptic inhibition from the dominant cell, but the membrane potential of the dominant cell stays constant for the duration of dominance, since there are no slow currents to cause it to decrease, and it is not under any synaptic influence from the inhibited cell. Thus dominant/inhibited transitions can only occur when the inhibited cell crosses the synaptic threshold. In our model, raising $b$ is analogous to lowering the synaptic threshold, and from Equation 27 we see that increasing $b$ results in an increase in frequency, as we would expect for the synaptic release mode of oscillation.

Thus oscillation via synaptic depression is qualitatively different from oscillation via intrinsic adaptation: synaptic depression oscillations occur only via a single mode, the synaptic escape mode, whereas intrinsic adaptation oscillations can occur via either the synaptic release or synaptic escape modes. Furthermore, this has immediately relevant functional consequences: The frequency of oscillation in an intrinsic adaptation RI circuit can either increase or decrease in response to increased tonic excitation, whereas the frequency of a synaptic depression RI circuit can only increase.

\section{Relation to previous work}

The idea that synaptic depression can lead to oscillations in an RI circuit is not novel. It was suggested in some of the earliest work on spinal CPGs in mammals (Graham Brown 1911) and since then has been considered as one of a handful of 'standard' mechanisms for generating oscillations in such a circuit (Reiss 1962, Perkel and Mulloney 1974, Friesen 1994, Ermentrout 1995).

Outside of their role in motor CPGs, RI circuits have also been considered as a possible mechanism underlying various kinds of perceptual alternations, such as binocular rivalry and Marroquin patterns (Matsuoka 1984, Blake 1989, Mueller 1990, Wilson, Krupa and Wilkinson 2000). Synaptic depres- 
sion has also been studied in connection with oscillations in larger networks. A model of rhythmic bursting in developing chick spinal cord relies upon synaptic depression of excitatory synapses on two separate time scales, one being that of the within-burst oscillations and the other being that of the inter-burst period (Tabak, Senn, O'Donovan and Rinzel 2000). Another recent model shows that large networks of randomly connected neurons with nonlinear synapses (in this case, synapses which both depress and facilitate) can spontaneously generate 'population bursts': times at which nearly all cells emit a spike, and do so in near-synchrony (Tsodyks, Uziel and Markram 2000).

However, none of the above work examines the case of two cells with depressing reciprocal inhibition in an analytically tractable model. As a result, none is able to derive simple expressions for the oscillatory regime, period, and amplitude of the resulting oscillations. Reiss (1962) considers a model similar to ours, but with spiking neurons that are not amenable to analysis. Matsuoka (1984) considers a model that is amenable to analysis, but incorporating a form of intrinsic adaptation rather than synaptic depression. Hence, this paper provides a novel analysis of a form of neuronal bursting. 


\section{References}

Abbott, L. F., Varela, J. A., Sen, K. and Nelson, S. B. (1997). Synaptic depression and cortical gain control, Science 275(5297): 220-4.

Arshavsky, Y. I., Deliagina, T. G., Orlovsky, G. N., Panchin, Y. V., Popova, L. B. and Sadreyev, R. I. (1998). Analysis of the central pattern generator for swimming in the mollusk clione, Annals of the New York Academy of Sciences 860(5): 51-69.

Blake, R. (1989). A neural theory of binocular rivalry, Psychological Review 96(1): 145-67.

Brodfuehrer, P. D., Debski, E. A., O'Gara, B. A. and Friesen, W. O. (1995). Neuronal control of leech swimming, Journal of Neurobiology $27(3)$ : 403-18.

Calabrese, R. L., Nadim, F. and Olsen, Ø. H. (1995). Heartbeat control in the medicinal leech: A model system for understanding the origin, coordination, and modulation of rhythmic motor patterns, Journal of Neurobiology 27(3): 390-402.

Ermentrout, G. B. (1995). Phase-plane analysis of neural activity, in M. Arbib (ed.), The Handbook of Brain Theory and Neural Networks, MIT Press, Cambridge, MA, USA, pp. 732-738.

Friesen, W. O. (1989). Neuronal control of leech swimming movements, in J. Jacklet (ed.), Neuronal and Cellular Oscillators, Marcel Dekker, New York.

Friesen, W. O. (1994). Reciprocal inhibition: A mechanism underlying oscillatory animal movements, Neuroscience and Biobehavioral Reviews 18(4): 547-53.

Galarreta, M. and Hestrin, S. (1998). Frequency-dependent synaptic depression and the balance of excitation and inhibition in the neocortex, Nature Neuroscience 1(7): 587-94.

Graham Brown, T. (1911). The intrinsic factors in the act of progression in the mammal, Proceedings of the Royal Society of London B84: 308-319. 
Grillner, S., Deliagina, T., Ekeberg, O., el Manira, A., Hill, R. H., Lansner, A., Orlovsky, G. N. and Wallén, P. (1995). Neural networks that coordinate locomotion and body orientation in lamprey, Trends in Neurosciences 18(6): 270-9.

Hopfield, J. J. (1984). Neurons with graded response have collective computational properties like those of two-state neurons, Proceedings of the National Academy of Sciences USA 81(10): 3088-92.

LoFaro, T., Kopell, N., Marder, E. and Hooper, S. (1994). Subharmonic coordination in in networks of neurons with slow conductances, Neural Computation 6(1): 69-84.

Mangan, P. S., Cometa, A. K. and Friesen, W. O. (1994). Modulation of swimming behavior in the medicinal leech. IV. Serotonin-induced alteration of synaptic interactions between neurons of the swim circuit, Journal of Comparative Physiology A 175(6): 723-36.

Manor, Y., Nadim, F., Abbott, L. F. and Marder, E. (1997). Temporal dynamics of graded synaptic transmission in the lobster stomatogastric ganglion, Journal of Neuroscience 17(14): 5610-21.

Matsuoka, K. (1984). The dynamic model of binocular rivalry, Biological Cybernetics 49(3): 201-8.

Mueller, T. J. (1990). A physiological model of binocular rivalry, Visual Neuroscience 4(1): 63-73.

Nadim, F., Manor, Y., Kopell, N. and Marder, E. (1999). Synaptic depression creates a switch that controls the frequency of an oscillatory circuit, Proceedings of the National Academy of Sciences of the United States of America 96(14): 8206-11.

Perkel, D. H. and Mulloney, B. (1974). Motor pattern production in reciprocally inhibitory neurons exhibiting postinhibitory rebound, Science 185(146): 181-3.

Reiss, R. F. (1962). A theory and simulation of rhythmic behavior due to reciprocal inhibition in small nerve nets, AFIPS Spring Joint Computer Conference, Vol. 21, National Press, San Francisco, California, USA, pp. 171-194. 
Roberts, A., Soffe, S. and Perrins, R. (1997). Spinal networks controlling swimming in hatchling Xenopus tadpoles, in P. Stein, S. Grillner, A. Selverston and D. Stuart (eds), Neurons, networks, and motor behavior, MIT Press, Cambridge, pp. 83-90.

Rowat, P. F. and Selverston, A. I. (1997). Oscillatory mechanisms in pairs of neurons connected with fast inhibitory synapses, Journal of Computational Neuroscience 4(2): 103-27.

Selverston, A. and Moulins, M. (1987). The Crustacean Stomatogastic System, Springer, Berlin.

Skinner, F. K., Kopell, N. and Marder, E. (1994). Mechanisms for oscillation and frequency control in reciprocally inhibitory model neural networks, Journal of Computational Neuroscience 1(1-2): 69-87.

Tabak, J., Senn, W., O’Donovan, M. J. and Rinzel, J. (2000). Modeling of spontaneous activity in developing spinal cord using activitydependent depression in an excitatory network, Journal of Neuroscience 20(8): 3041-56.

Taylor, A., Cottrell, G. W. and Kristan, Jr., W. B. (2000). A model of the leech segmental swim central pattern generator, Neurocomputing 32-33: 573-84.

Tsodyks, M., Uziel, A. and Markram, H. (2000). Synchrony generation in recurrent networks with frequency-dependent synapses, Journal of Neuroscience $\mathbf{2 0}(1)$ : $\mathrm{RC} 50$.

Tsodyks, M. V. and Markram, H. (1997). The neural code between neocortical pyramidal neurons depends on neurotransmitter release probability, Proceedings of the National Academy of Sciences of the United States of America 94(2): 719-23.

Wang, X.-J. and Rinzel, J. (1992). Alternating and synchronous rhythms in reciprocally inhibitory model neurons, Neural Computation 4: 44-97.

Wilson, H. R. and Cowan, J. D. (1972). Excitatory and inhibitory interactions in localized populations of model neurons, Biophysical Journal 12(1): $1-24$. 
Wilson, H. R., Krupa, B. and Wilkinson, F. (2000). Dynamics of perceptual oscillations in form vision, Nature Neuroscience 3(2): 170-6. 